\title{
Evaluation of Bismuth Oxide Nanoparticles as Radiosensitizer for Megavoltage Radiotherapy
}

\author{
Noor Nabilah Talik Sisin", Safri Zainal Abidin", Muhammad Amir Yunus ${ }^{*}$, Hafiz M. Zin*, \\ Khairunisak Abdul Razak ${ }^{+}$, Wan Nordiana Rahman ${ }^{\#}$

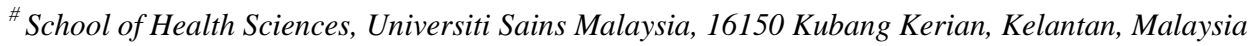 \\ E-mail:nabilah.talik@gmail.com; wandiana@usm.my \\ *Advanced Medical and Dental Institute, Universiti Sains Malaysia, 13200 Bertam, Penang, Malaysia \\ E-mail:safri@usm.my; amiryunus@usm.my; hafiz.zin@usm.my \\ ${ }^{+}$School of Materials and Mineral Resources Engineering, Universiti Sains Malaysia, 14300 Nibong Tebal, Penang, Malaysia \\ E-mail: khairunisak@usm.my
}

\begin{abstract}
Metal-based nanoparticles such as gold, silver, platinum, and bismuth have been widely investigated for radiotherapeutic application. Basic understanding of the cellular interaction of the nanoparticles with the biological materials is crucial to ensure future clinical use. In this study, the cytotoxicity, cellular uptake, and generation of reactive oxygen species (ROS) induced by BiONPs were investigated prior elucidating the feasibility of BiONPs for radiotherapy application using megavoltage photon and electron beams. The BiONPs of diameter sizes $60,70,80$ and $90 \mathrm{~nm}$ at concentrations within a range of 0.5 to $0.00005 \mathrm{mMol} / \mathrm{L}$ were tested on MCF-7, MDA-MB-231, and NIH/3T3 cells lines. The cytotoxicity results exhibit minimal cell death constituting less than $20 \%$ of mortality on average. The ROS generation by BiONPs alone is found to be negligible as the ROS levels were slightly lower and higher than $100 \%$ of positive control. The increment of cellular nanoparticles uptake from a range of $1.50 \%$ to $34.10 \%$ indicates that BiONPs were internalized and bound to the surface of the cells. Sequencing from the results, $60 \mathrm{~nm}$ BiONPs are found to be the most suitable to be applied as a radiosensitizer in radiotherapy. Sensitization enhancement ratio (SER) quantified on MCF-7 cells demonstrated the highest enhancement from the highest concentration of BiONPs with SER of 2.29 and 1.42, for $10 \mathrm{MV}$ photon beam and $6 \mathrm{MeV}$ electron beam, respectively. In contrast to ROS production without radiation, the ROS induced from radiotherapy beams were found to be dose-dependent and play significant roles in radiosensitization effect. In conclusion, BiONPs could improve clinical radiotherapy, and further radiobiological characterization is crucial for future clinical translation.
\end{abstract}

Keywords - bismuth oxide nanoparticles; cytotoxicity; reactive oxygen species; radiotherapy.

\section{INTRODUCTION}

Materials in nanometer scales have long existed in our nature. However, only recently, that systems and technology have advanced towards nanoscales application in many fields, including medicine. Nanosized materials such as nanoparticles (NPs) are defined as an aggregation of matter with a radius of not more than $100 \mathrm{~nm}$ [1]. Metal NPs such as gold started to be used in biomedical applications due to their intriguing biological properties [2]. Currently, nanotechnology has hugely contributed in prevention, diagnostics, and treatments of diseases, especially in drug delivery, tissue engineering, magnetic resonance imaging, cancer therapy, tissue repair, and cellular therapy [3], [4].

In radiotherapy, therapeutic nanoparticles are the growing trends in research, and the NPs with high atomic numbers $(\mathrm{Z})$ have been extensively investigated for their excellent radiosensitizing effects in cancer treatment. High dose of radiation in eliminating cancer cells usually affected the surrounding healthy tissue and induced complication [5]. The presence of NPs in a tumor would help local absorption of the radiation energy and concentrate more dose at the target site, thus contributed to the DNA damage of the cancer cells [6].

In a clinical setting nowadays, radiosensitizers used are usually in the form of chemotherapy drugs such as cisplatin, capecitabine, and 5-fluorouracil [7]-[9]. However, one of the major concerns is their cytotoxicity effects on healthy normal cells. The drug used would be widely taken up by both normal and cancer tissues, and the tissues would be affected by both chemotherapy and radiotherapy [8]. An in vivo research demonstrated that radiotherapy, in combination with cisplatin had caused significant systemic toxicities [7]. 
Also, a few clinical cases reported that capecitabine and 5fluorouracil could cause hand-foot syndrome, diarrhea, lung hemorrhage, as well as life-threatening toxicity [10], [11].

Metal NPs have begun to be considered as safer alternatives as radiosensitizers. Since NPs did not dissolve in solution, the entry to normal capillaries and vasculature are limited, and the half-life of NPs in the human body is longer than the usual drugs [8]. Carbon black, zinc dioxide, silicon dioxide and single-wall carbon nanotube NPs with sizes range between 8 to $21 \mathrm{~nm}$ were tested on primary fibroblast cell BALB/3T3 demonstrated low cytotoxic properties (cell viability more than $80 \%$ ) when treated with concentrations of 5 and $10 \mu \mathrm{g} / \mathrm{ml}$ [12]. Even a $60 \mu \mathrm{g} / \mathrm{ml}$ of bismuth subsalicylate concentration had shown only $6 \%$ of cytotoxicity on gingival fibroblast HGF-1 cells [13].

NPs of different types have been preclinically investigated for their potential application in radiotherapy. Gold NPs are one of the most widely studied nanoparticles found to enhance the radiation dose at kilovoltage [14], clinical megavoltage photon beam [15], [16], synchrotron microbeam [6] and ${ }^{192}$ Ir brachytherapy [17]. Supporting the inert and biocompatible properties of Gold NPs, cytotoxicity assessment indicate more than $75 \%$ of cell viability depending on the NPs concentrations and the cell types [3], [14]. Apart from gold NPs, gadolinium oxide NPs were tested on CT26 cells using $50 \mathrm{keV}$ synchrotron radiation and had been found to enhance ROS production, suggesting the subsequent dose enhancement effect [18]. Moreover, iron oxide NPs expressed the dose enhancement factor (DEF) of 2.5 when tested on T24 bladder cancer cells using a clinical $10 \mathrm{MV}$ photon beam [19].

While most studies focus on engineered metal NPs involved gold, bismuth has also begun to be investigated as an alternative material due to its low toxicity and costeffective attributes [20]. Bismuth and its complexes are found to have many properties such as antibacterial [13], antiproliferative, antimicrobial [21] and high radiation absorption coefficient that make it suitable as contrast agents in X-ray computed tomography for medical imaging [20]. Bismuth oxide NPs (BiONPs) have been introduced as a radiosensitizer because the presence of bismuth may trigger additional retention, absorption, and scattering of the radiation at the cancer site [22], and thus demonstrated a higher enhancement of the dose, in comparison to other types of NPs [23]. Cytotoxicity effects have been reported in previous literature indicating $50 \%$ of cells inhibition concentration values $\left(\mathrm{IC}_{50}\right)$ caused by BiONPs on HepG2 liver, NRK-52E kidney, Caco-2 colorectal and A549 lung cell lines [24]. Another study conducted on Chang liver cells found that the BiONPs cytotoxicity was associated with the temperature during BiONPs synthesis [25]. In regards to their attractive biocompatibility profile, the potential of BiONPs as radiosensitizer has been investigated in vitro, in vivo as well as in silico and phantoms studies which presented interesting results [26]-[28]. The researches above though did not investigate the applicability of the BiONPs on the breast cancer radiotherapy, and this report will be the first empirical precedent to apply BiONPs for clinical megavoltage beams.

In this study, we intend to investigate the fundamental biological characteristic of BiONPs such as cytotoxicity, cellular uptake as well as reactive oxygen species (ROS) generation on normal and breast cancer cells, as the preliminary analysis for the development of novel non-toxic radiosensitizer. Later we quantify the radiosensitization effects and ROS production of BiONPs after treatment with clinical radiotherapy beams to observe the actual feasibility of the BiONPs application in breast cancer radiotherapy.

\section{MATERIALS AND METHODS}

\section{A. BiONPs Preparation}

The experiments were conducted using four different sizes of rod-shaped BiONPs which are 60, 70, 80 and $90 \mathrm{~nm}$. We chose the sizes of less than $100 \mathrm{~nm}$ following the definition of nanomaterials by Stewart [26]. On the other hand, there are research that studied the sizes of nanoparticles with high gap such as $10 \mathrm{~nm}$ over $300 \mathrm{~nm}$ [29], $25 \mathrm{~nm}$ over $50 \mathrm{~nm}$ [30], and 10 over $100 \mathrm{~nm}$ [31]. Hence our study would like to clarify the influence of the small difference between the sizes. Thus, we used these 4 sizes. The BiONPs of various sizes were synthesized using the hydrothermal method and characterized as reported in previous literature [32], [33]. The BiONPs were diluted with Dulbecco's Modified Eagles Medium (DMEM, Gibco, USA) and stored in $4^{\circ} \mathrm{C}$ for in-vitro study.

\section{B. Cell culture: $M C F-7, M D A-M B-231$, and NIH/3T3}

MCF-7 (human breast adenocarcinoma), MDA-MB-231 (human mammary gland carcinoma) and NIH/3T3 (mouse embryonic fibroblast) cell lines were used in the in-vitro study. All types of cell lines were cultured in Dulbecco's Modified Eagles Medium (DMEM, Gibco, USA), supplemented with $5 \%$ of fetal bovine serum (FBS) (Gibco, USA) and $1 \%$ penicillin-streptomycin (Gibco, USA). Subculturing of the cell lines was performed by detaching the adherent cells using $0.025 \%$ trypsin-EDTA (Gibco, USA), and the cells were maintained by routine passage every two to three days. Phosphate Buffered Saline (PBS) (Gibco, UK) was used to wash the debris and floated dead cells in the flasks. Cell incubator humidified with $5 \% \mathrm{CO}_{2}$ in $37^{\circ} \mathrm{C}$ was used to keep the cells grown.

\section{In Vitro Cytotoxicity Effects}

1) Cell Viability Measurement with Prestoblue Assay (Pre-Irradiation): The cellular effects of the BiONPs on cell viability without irradiation were measured using Prestoblue assay, as a preliminary study to infer the significance of BiONPs on the cells. The study was conducted by culturing approximately $2 \times 10^{4}$ of cells per $\mathrm{ml}$ in 96 wells plates until reaching 70 to $80 \%$ confluency. The cells were then treated with $0.5,0.05,0.005,0.0005$ and $0.00005 \mathrm{mMol} / \mathrm{L}$ of BiONPs for 24, 48 and 72 hours respectively. Measurements of the viability of the cells were performed by replacing the cells media with $90 \mu \mathrm{l}$ of fresh media and adding $10 \mu \mathrm{l}$ of Prestoblue reagent (Invitrogen, USA). The cells were incubated with Prestoblue for around 4 hours. The fluorescence was measured using a microplate reader at 535 $\mathrm{nm}$ excitation and $615 \mathrm{~nm}$ emission wavelength.

2) ROS induced by BiONPs Measurement (PreIrradiation): The ROS produced by BiONPs were measured according to the protocol reported in previous literature [12], 
[34], as a pilot study to analyze the appropriate use of BiONPs. The $5 \times 10^{4}$ cells per ml were cultured in 96 wells plates and incubated overnight until it reached 50-70\% confluency. After incubation, the cells were treated with BiONPs, washed with PBS, and $10 \mu \mathrm{l}$ of 2', 7'dichlorodihydrofluorescein diacetate $\left(\mathrm{DCFH}_{2}-\mathrm{DA}\right.$, SigmaAldrich) diluted in DMSO was added in each well. Then, the cells with light-sensitive $\mathrm{DCFH}_{2}$-DA were incubated at 37 ${ }^{\circ} \mathrm{C}$ in the dark for around 60 minutes. The fluorescence was then determined at $485 \mathrm{~nm}$ excitation and $530 \mathrm{~nm}$ emission using microplate reader, immediately after treatment, 3.5 hours, 6 hours, and 24 hours.

\section{BiONPs Uptake Measurement}

The BiONPs uptake was measured using flow cytometrybased on a previously established method [35], as a prelude investigation to pre-determine the relevancy of the BiONPs application. Cells were counted to be $1 \times 10^{4}$ cells per ml and then were cultured in 6-well plates and incubated at 37 ${ }^{\circ} \mathrm{C}$ for 24 hours. After incubation, the cells were washed gently with PBS and treated with $0.5 \mathrm{mMol} / \mathrm{L}$ of $60 \mathrm{~nm}$ BiONPs for 24 hours. In this study, we only measured the uptake of $60 \mathrm{~nm}$ BiONPs. The cells were rewashed with PBS, before being trypsinized from the plates to prepare the cell suspension in flow cytometry tubes. $1 \mathrm{ml}$ of culture medium was added to the cells suspension to neutralize the trypsin. The cells were centrifuged at $1500 \mathrm{rpm}$ for $5 \mathrm{~min}$ and washed with PBS twice. Lastly, the cells were suspended in PBS with a maximum volume of $400 \mu \mathrm{l}$ and left on ice until the time for analysis. Flow cytometry was performed using a blue fluorescent filter (FACS Canto II, BD Biosciences, US).

\section{E. Quantification of Radiosensitization Effects for Megavoltage Radiotherapy}

1) Sensitization Enhancement Ratio Measurement: The effects of the megavoltage radiotherapy in combination with BiONPs were evaluated on MCF-7 cells and $60 \mathrm{~nm}$ BiONPs. MCF-7 cell samples were prepared with and without BiONPs of $60 \mathrm{~nm}$ size with the concentration of $0.05,0.25$, and $0.05 \mu \mathrm{Mol} / \mathrm{L}$. The samples were exposed to radiation doses from 0 to $10 \mathrm{~Gy}$ irradiated with 6 and $10 \mathrm{MV}$ photon beams, as well as 6 and $12 \mathrm{MeV}$ electron beams generated from a medical linear accelerator (Elekta Synergy, Sweden) at Advanced Medical and Dental Institute, Universiti Sains Malaysia. The irradiation was done at a dose rate of 599 MU/min. Clonogenic assays determined cell survival after 5 days. The cell survival for samples with and without BiONPs was then plotted and fitted according to the linearquadratic (LQ) model using OriginPro 8.5 software. The parameters from the LQ formula given by equation 1 were analyzed.

$$
S=\exp ^{-(\alpha D+\beta D 2)}
$$

In the equation, $\mathrm{S}$ is the survival fraction and $\mathrm{D}$ is the dose in Gray. The model represented by the linear component $[\exp (-\alpha D)]$ and quadratic component $\left[\exp \left(-\beta \mathrm{D}^{2}\right)\right]$ where $\alpha$ indicate single hit double-strand break of two chromosomes and $\beta$ show double hits that induce double-strand break of two chromosomes.

The radiosensitization effects were quantified by calculating the sensitization enhancement ratio (SER). Equation 2 shows the SER calculation:

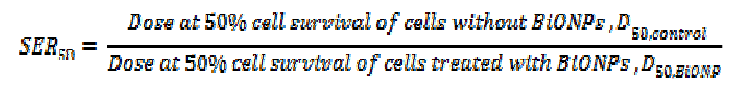

2) ROS induced by BiONPs Measurement (PostIrradiation): Measurements of ROS caused by BiONPs under irradiation with megavoltage radiotherapy beam were performed on MCF-7 cells and $60 \mathrm{~nm}$ BiONPs. ROS was evaluated by using cells cultured in 96 well plates (approximately $1 \times 10^{4}$ cells per well) incubated for 24 hours. The cells were then treated with BiONPs with different concentrations $(0.5,5.0$, and $50.0 \mu \mathrm{Mol} / \mathrm{L})$ and DCFH-DA reagent was added in each well to make up a final concentration of $50 \mu \mathrm{M}$. The cells were irradiated using a similar irradiation setup with SER measurement. The ROS generations after the irradiation were measured using microplate reader (FLUOstar Omega, BMG Labtech, German) with an excitation of $485 \mathrm{~nm}$ and an emission of $520 \mathrm{~nm}$ immediately after irradiation.

\section{F. Statistical Analysis}

The data were expressed as the mean \pm standard error of the mean (SEM) and mean \pm standard deviation (SD). All graphs and statistical tests were plotted and performed using OriginPro 2018 software (OriginLab Corporation, US).

\section{RESULT AND DISCUSSION}

\section{A. In Vitro Cytotoxicity Effects}

1) Cell Viability Measurement with Prestoblue Assay (Pre-Irradiation): Cytotoxicity effects of 60, 70, 80 and 90 $\mathrm{nm}$ BiONPs of different concentration is shown in Fig. 1. All cells show more than $80 \%$ cell viability when treated with all different sizes and concentrations of BiONPs. However, the NIH/3T3 and MDA-MB-231 cells show more than $20 \%$ of cell death at 48 and 72 hours when treated with 0.5 $\mathrm{mMol} / \mathrm{L}$ of $70,80,90 \mathrm{~nm}$ of BiONPs, as depicted in Fig. 1 $(\mathrm{B}, \mathrm{C}, \mathrm{D}, \mathrm{J}$, and L). Treatment with 0.05 and $0.5 \mathrm{mMol} / \mathrm{L}$ of 70, 80, $90 \mathrm{~nm}$ BiONPs on MCF-7 cells as shown in Fig. 1 (F) also indicate cell death more than $20 \%$ even at 24 hours incubation. Meanwhile, $60 \mathrm{~nm}$ BiONPs are biocompatible on cancer and normal cells for all concentrations tested after 24, 48 and 72 hours. The results presented more than $80 \%$ cell viability against both normal fibroblast and breast cancer cell lines, in Fig. 1 (A, E and I). The $60 \mathrm{~nm}$ BiONPs at 0.005 $\mathrm{mMol} / \mathrm{L}$ of concentration particularly are found to induce less toxicity in comparison to other sizes and concentration. Thus, the $60 \mathrm{~nm}$ BiONPs is considered the optimal size for potential application as a radiosensitizer. 
A. $60 \mathrm{~nm}$ on NIH/3T3

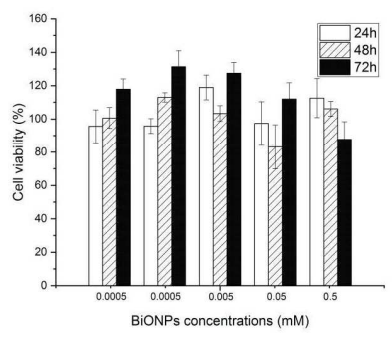

E. $60 \mathrm{~nm}$ on MCF-7

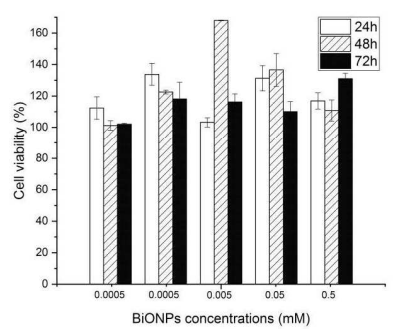

I. $60 \mathrm{~nm}$ on MDA-MB-231

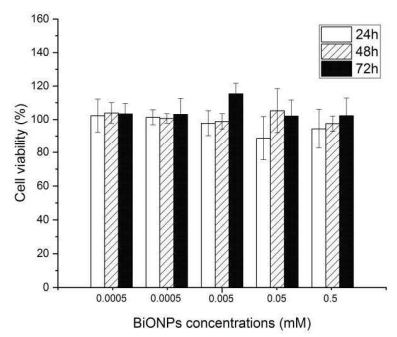

B. $70 \mathrm{~nm}$ on $\mathrm{NIH} / 3 \mathrm{~T} 3$

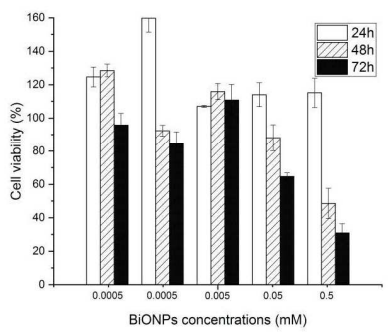

F. $70 \mathrm{~nm}$ MCF-7

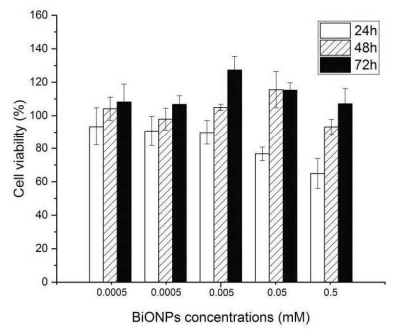

J. $70 \mathrm{~nm}$ on MDA-MB-231

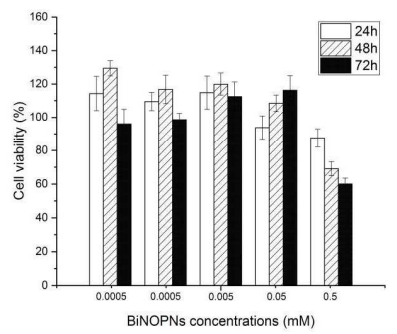

C. $80 \mathrm{~nm}$ on NIH/3T3

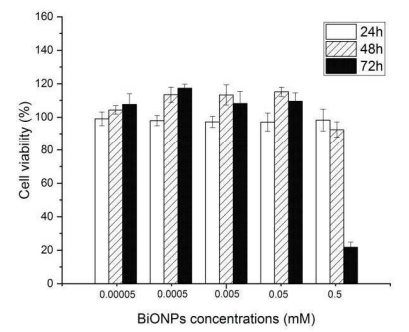

G. $80 \mathrm{~nm}$ on MCF-7

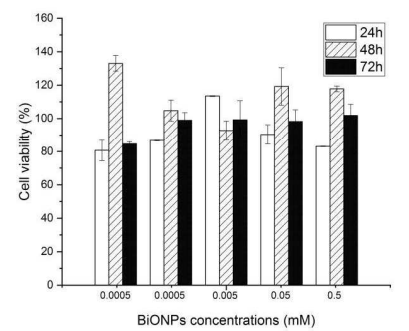

K. $80 \mathrm{~nm}$ on MDA-MB-231

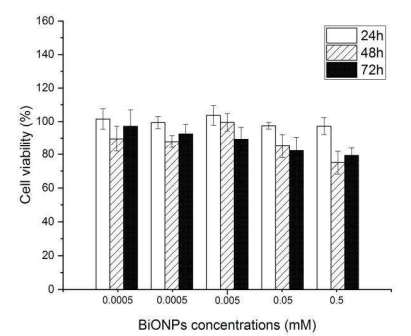

D. $90 \mathrm{~nm}$ on $\mathrm{NIH} / 3 \mathrm{~T} 3$

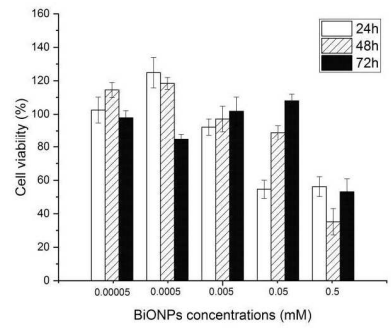

H. $90 \mathrm{~nm}$ on MCF-7

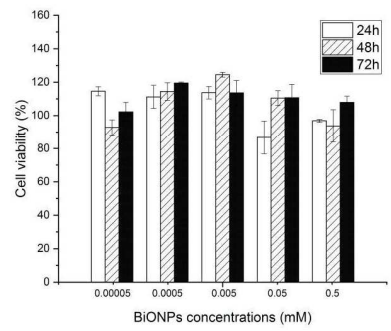

L. $90 \mathrm{~nm}$ on MDA-MB-231

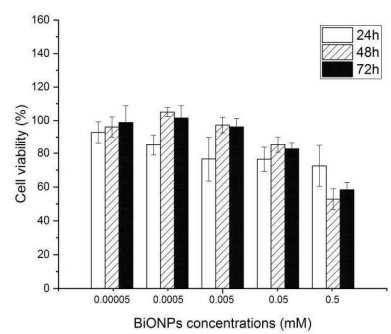

Fig. 1 Cytotoxicity assay of different sizes $(60,70,80$, and $90 \mathrm{~nm})$ and concentrations $(0.00005,0.0005,0.005,0.05$, and $0.5 \mathrm{mM})$ of BiONPs-treated cells. (A) (B) (C) and (D) BiONPs treatment on NIH/3T3 cells. (E), (F), (G) and (H) BiONPs treatment on MCF-7 cells. (I), (J), (K) and (L) BiONPs treatment on MDA-MB-231 cells. The x-axis depicts the concentrations, in mM. Y-axis shows the percentage of active cells. Legends indicate the hours of BiONPs treatment incubations, in hours. Each error bar represents the standard error of mean (SEM).

The results demonstrated the cytotoxicity dependency on BiONPs size. Smaller nanoparticles (NPs) have a larger surface-area-to-volume ratio and hence induced higher surface reactivity and cytotoxicity [36]. Several studies also found that cytotoxicity of several types of NPs was sizedependent. Studies conducted on large ranges NPs sizes, such as 10 to $300 \mathrm{~nm}$ silica and gold NPs [29], 50 to $80 \mathrm{~nm}$ BiONPs [26], 3 to $50 \mathrm{~nm}$ gold NPs [30], 10 to $100 \mathrm{~nm}$ silver NPs [31] indicate effects of size on cytotoxicity. A study showed that $50 \mathrm{~nm}$ NPs were only localized in the cytoplasm, not the nucleus. Thus the low toxicity as the membrane damages was not high [30]. These results were in agreement with our current findings in which BiONPs in the diameter sizes of $60,70,80$ and $90 \mathrm{~nm}$ could only cause minimal cytotoxicity. However, the cytotoxicity of BiONPs is also dependent on incubation time and cell types as observed in this study.

Prestoblue reagent was used for the cytotoxicity study, as it was considered as a fast and non-toxic assay [37], [38]. The detection of cell viability depends on the functions of the mitochondrial enzymes within the live cells which reacted with the rezasurin compound [37]-[39] The fluorescent reduced formed of rezasurin changed the color of the cell media and could be measured by both fluorescence and absorbance [40]. A quantitative study stated that Prestoblue gives a stronger fluorescent signal than Alamar blue assay[41]. Prestoblue assay is a ready-to-use assay as it only needed one single step compared to MTT and LDH release assays [39]. It is also more sensitive compared to the MTT assay as the latter required a longer time to be able to detect the cells as well as toxic to the cells [38], [40].

2) ROS induced by BiONPs Measurement (PreIrradiation): The presence of oxidative stress yielded by the NPs in the cell would produce the free radicals that will possibly attack the DNA in the cells [12]. Fig. 2 shows the intercellular generation of ROS due to the presence of BiONPs. After treatment with different sizes and different concentrations of BiONPs on the NIH/3T3 cells, it is observed that the formation of ROS is not affected by the size and concentration of NPs (Fig. 2A and 2D). Nevertheless, the ROS measured at 24 hours of treatment are found to be lower than at 6 hours in normal NIH/3T3 cells. It may suggested that ROS decrease after 6 hours and this could be the reason of the minimal toxicities caused by the BiONPs on NIH/3T3 cells after 24, 48, and 72 hours periods (Fig. 1). This finding is supported by Zhu et al. in which they hypothesized that the upregulated endogenous ROS production in normal cells due external stress would not 
exceed the toxic threshold of ROS [42]. The antioxidant enzymes within the cells such as catalase (CAT), superoxide dismutase (SOD), and gluthathione peroxidase (GPx) would detoxify the ROS [43]-[45]. Thus, the redox activity happened and lowered back the ROS level [42].

Furthermore, after treatment with different sizes and different concentrations of BiONPs on MCF-7 cells, the
DCF fluorescence elevated up to 3.5 hours, before it decreased afterward. In Fig. 2 (B), the ROS continued to deplete as time increase, however, in Fig. 2 (E), the percentage of ROS started to increase again at 24 hours. The induced ROS formation is in a time-dependent manner. The previous study had also noted that MCF-7 cells could cause time-dependent cytotoxicity due to ROS production [4].
A. ROS on NIH/3T3

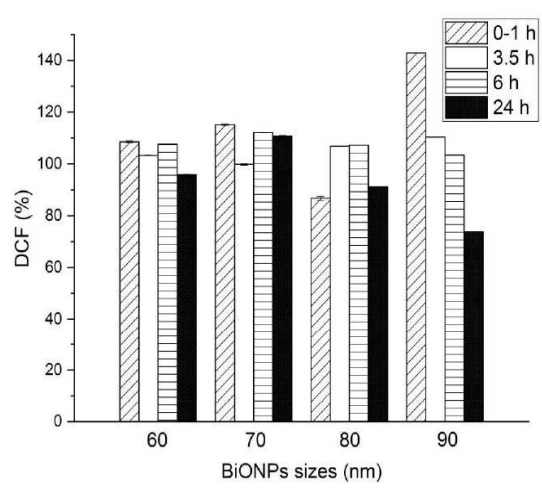

D. ROS on NIH/3T3

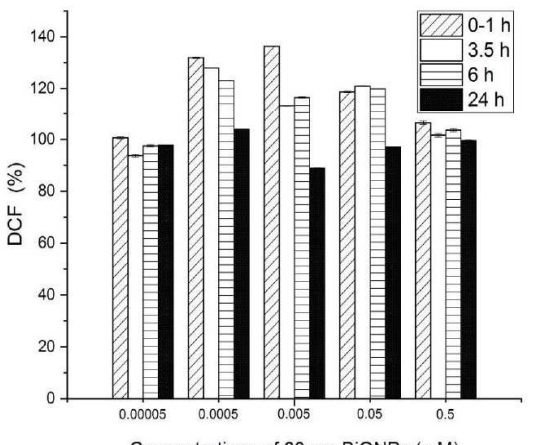

B. ROS on MCF-7

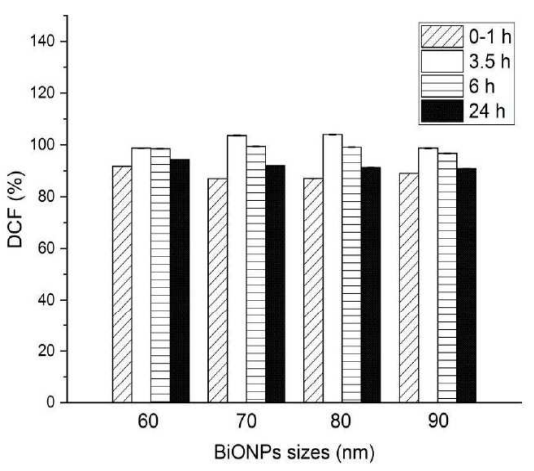

E. ROS on MCF-7

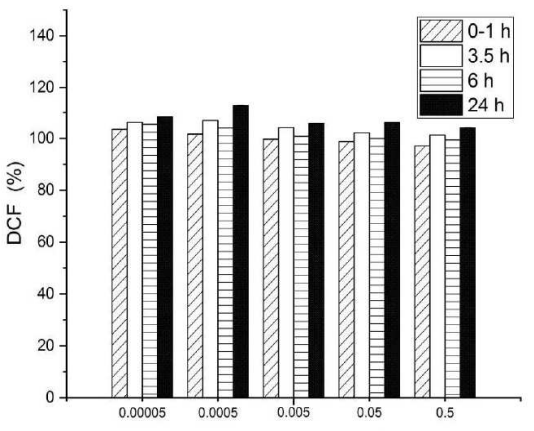

Concentrations of $60 \mathrm{~nm}$ BiONPs (mM)
C. ROS on MDA-MB-231

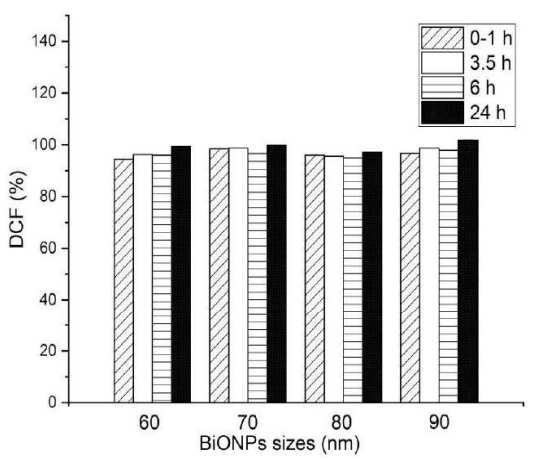

F. ROS on MDA-MB-231

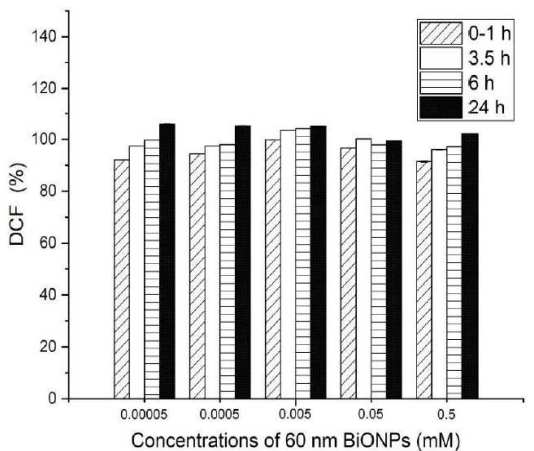

Fig. 2 ROS measurement of BiONPs on NIH/3T3, MCF-7, and MDA-MB-231 cells. The treated cells were compared to control cells treated with DCFH-DA only (positive control), $100 \%$ DCF fluorescent. Graphs A, B and C were based on $0.5 \mathrm{mM}$ of different BiONPS sizes, while graphs D, E and F were based on different concentrations of $60 \mathrm{~nm}$ BiONPs. Each error bar represents the standard error of mean (SEM).

On the other hands, the ROS generation in MDA-MB-231 cells generally occurred in the same increasing pattern from 0 to 24 hours. The percentages of DCF detected were also within the range of 91 to $106 \%$, though with different sizes and different concentrations. Most of the cells either induced ROS in lower percentage or slightly higher than that of positive control (100\%).

For this study, we deduced that the ROS formation did not play a critical role in the cytotoxicity of the BiONPs with slightly different diameter sizes. The findings in the present study are in agreement with a previous study which implied that any mechanism caused by the ROS generation would not significantly affect the DNA of the cells and thus, ROS generation was only the secondary force to the onset of cytotoxicity [46]. Another research also had identified bismuth oxide nanoceramics as ROS scavengers depending on the NPs production process [26]. Meanwhile, a study on erythrocytes revealed that a bismuth-based compound has antioxidant activity and offered protection against the toxicity by other particles [47]. Even though the latest investigation showed BiONPs could induce significantly high ROS but the lowest BiONPs concentration used was 50 $\mu \mathrm{g} / \mathrm{ml}$ [48], while our study only used $0.5 \mu \mathrm{M}$ or approximately $0.233 \mu \mathrm{g} / \mathrm{ml}$ of BiONPs as the highest concentration. Hence, it is suggested that the treatment of BiONPs on the cells in our study did not induce high oxidative stress and could be considered as biocompatible with the cells.

\section{B. BiONPs Uptake Measurement}

Fig. 3 illustrates the localization of $60 \mathrm{~nm}$ of BiONPs treatment on cells, obtained via flow cytometric analysis. The method of nanoparticle uptake detection by flow cytometry was already established and widely used in many studies. It is considered as a robust high throughput method and required only a couple of seconds for a maximum of 10 to 50 thousands cells [35], [36], [49]. The preparation time is more simple and fast, while the results generated are in high quality and statistically relevant as the cells are individually analyzed in comparison to the transmission electron microscope and inductively coupled plasma mass spectrometry [35], [49], [50]. The use of flow cytometry for 
nanoparticle internalization also could simultaneously explored other parameters such as cell cycles, cells apoptosis, cells necrosis, DNA damages, ROS generation, as well as cell types differentiation, in which appropriate fluorescent probes are needed respectively [35], [36], [49], [51]. In Fig. 3, each box is gated by side scattering (SSC) and forward scattering (FCS). The SSC and FSC represent the granularity and the size of the cells, respectively [26], [49].
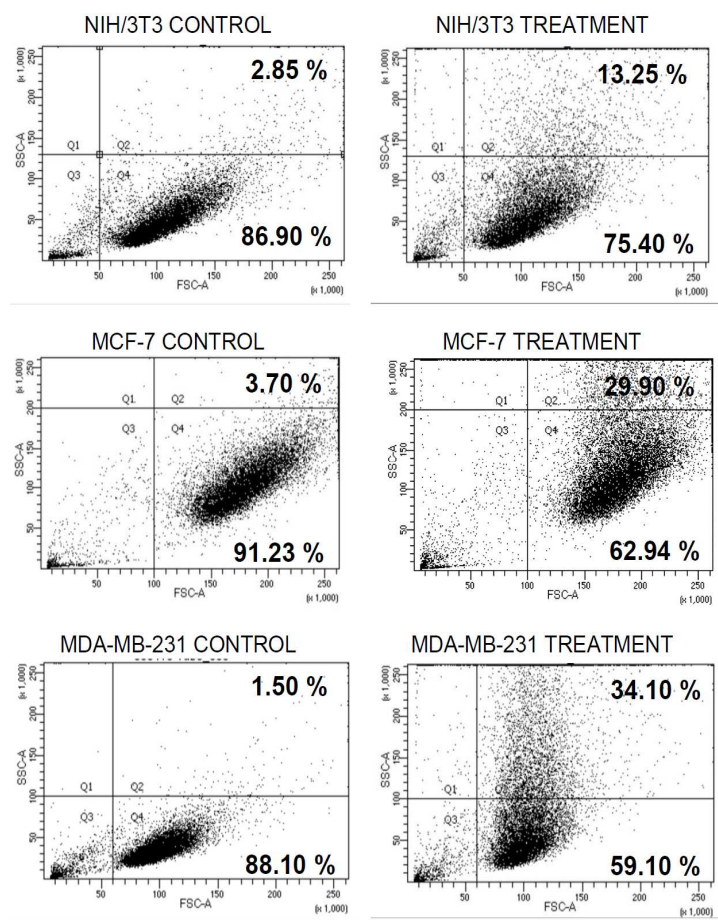

Fig. 3 Cellular uptakes due to the treatment with $0.5 \mathrm{mMol} / \mathrm{L}$ of $60 \mathrm{~nm}$ BiONPs on 3 types of cell lines; NIH/3T3, MCF-7 and MDA-MB-231 cells. Increments of percentage in quadrant Q2 are observed indicating the occurrence of nanoparticle uptakes.

The Q1 and Q3 quadrants were distinguished as a dead cell or cell debris population, while Q2 and Q4 quadrants portrayed live cells population. Previous studies stated that the uptake of the NPs would not influence the FSC, but it would depend on the size of the NPs [26], [52], [53].

The NIH/3T3, MCF-7 and MDA-MB-231 cells that were treated with $0.5 \mathrm{mMol} / \mathrm{L}$ of $60 \mathrm{~nm}$ BiONPs showed a substantial increase in granularity, which leads to a higher percentage at Q2 population (13 to $35 \%$ ), compared to control cells ( 1 to $4 \%$ ). The rise of Q2 populations suggested that the BiONPs localized on the surface of the cells and had been internalized in the cells. In the previous study, the highest amount of cellular uptake of NPs ensued at the size of $50 \mathrm{~nm}$. NPs cellular uptake would decrease for larger NPs [38].

Incorporation of NPs within the cells will significantly induce cytotoxicity and could cause irreparable DNA damages [31], [53], [54]. NPs size plays essential roles in the cellular NPs uptake. In this study, $60 \mathrm{~nm}$ BiONPs are found to be biocompatible that could be due to that most of these sizes of BiONPs were mostly bound to the surface of the cells and a few NPs were internalized inside cells. This assertion can be associated with the minimal cytotoxicity and high biocompatibility of $60 \mathrm{~nm}$ of BiONPs on both healthy and breast cancer cells.

\section{Radiosensitization Effects for Megavoltage Radiotherapy}

1) Sensitization Enhancement Ratio Measurement: Given the primary purpose was to used BiONPs as a radiosensitizer with low toxicity, lower concentrations of 60 $\mathrm{nm}$ BiONPs $(0.05,0.25$, and $0.5 \mu \mathrm{M})$ were tested on MCF-7 cell. Fig. 4 depicts the cell survival curves for MCF-7 cells irradiated with both photon and electron beams. Generally, the presence of BiONPs during the radiotherapy had enhanced the radiosensitization effect of the radiation, thus lowers the survival of the cancer cells. The survival curves also indicate that the radiosensitization on the cells is dependent on the BiONPs concentration, beam quality, and radiation dose.
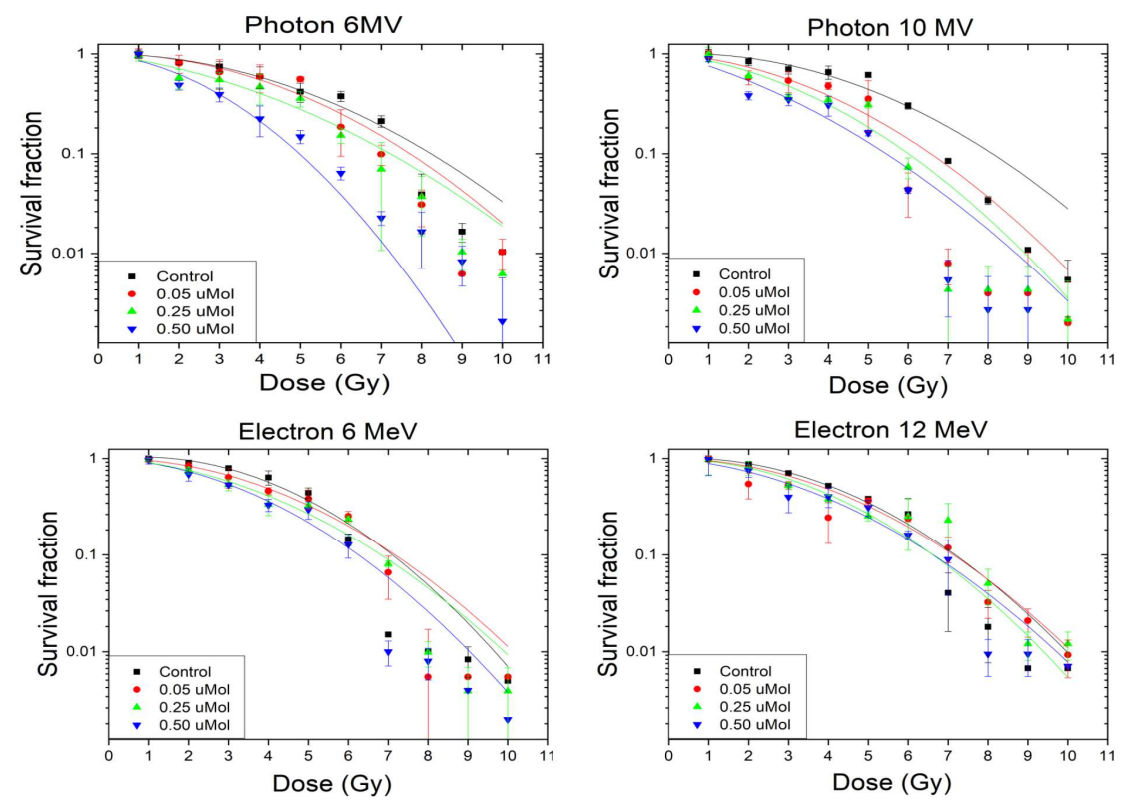

Fig. 4 Survival curves of MCF-7 cells with three concentrations of $60 \mathrm{~nm}$ of BiONPs, irradiated with $6 \mathrm{MV}$ and $10 \mathrm{MV}$ 
The effects are quantified as sensitization enhancement ratio (SER) values for photon and electron beams and tabulated in Table I. The increasing value of SER indicates the radiosensitization effects are dependent on BiONPs concentration and radiation energy. The concentration of 0.5 $\mu \mathrm{M}$ of BiONPs, which give the highest SER value for each energy, had caused the most cell death in this study with a lower radiation dose compared to the other BiONPs concentrations. These findings are supported by other studies on BiONPs [27], [28]. As a higher amount of BiONPs present, more radiation interactions might transpire against cancer cells. The penetration power also increases as the energy of the beam used were higher [28], [55].

A 3D phantoms study had demonstrated that the BiONPs was effective in enhancing the ionizing radiation dose of megavoltage $\mathrm{x}$-ray beams with an enhancement factor of 1.25 [27]. Besides, a Monte Carlo simulation of BiONPs irradiations had revealed a high SER range around 16 to 19 . The difference in the SER values was due to the difference in the concentration of BiONPs used. Nonetheless, the result from the present study can be correlated with the previous studies as mentioned earlier in which they predicted that the presence of BiONPs would increase the effective atomic numbers, absorption probabilities and radiation scattering [22], [28]. The internalized BiONPs reactions with the radiation beam would enhance the damaging effects on the DNA of cancer cells.

It is mentioned that interactions between radiation energies and high $\mathrm{Z}$ materials would initiate photoelectric absorption, Compton effect and pair productions [3]. The effects which mostly depends on the atomic numbers $\mathrm{Z}$ is the photoelectric effect [56]. High atomic numbers would elevate the photoelectric absorption, thus released more ions and free radicals, leading to cell damages and cell deaths [56]. However, previous works demonstrated that photoelectric interactions are not dominant for megavoltage beam, as higher energy would cause a higher Compton scattering [27], [57]. In the presence of high atomic number element, such as bismuth, the dominant interaction at the energy of more than $5 \mathrm{MeV}$ would be the electron-positron pair production [26]. These interactions would contribute to the breakage of the single and double strands of the DNA.

More importantly, the SER values of BiONPs radiosensitization from the present study were higher than aforementioned works on different metal NPs, such as gold NPs with DEF of 1.31 and 1.54 when using $10 \mathrm{MV}$ photon beam and brachytherapy respectively [15], [17], tagged gold NPs with DEF of 1.25, and combination of gold NPs with cisplatin with DEF of 1.14 at $225 \mathrm{kVp}$ using small animal irradiator [7]. The results from this study are also in close agreement with another BiONPs study on 9L gliosarcoma cells which presented SER of 1.25 and 1.48 for $125 \mathrm{kVp}$ and $10 \mathrm{MV}$ energies [23]. Future studies are currently underway to evaluate the radiosensitization effect of $60 \mathrm{~nm} \mathrm{BiONPs}$ using other radiation energy and beams.
Nevertheless, this study will open the pathways to decrease the mortality rate of breast cancer patients specifically. Enhancement of the radiation effects with the radiosensitizer would decrease the prescribed radiation doses to the patients. Therefore, the effect of radiation on nearby healthy organs might also be reduced and possibly lowered the likelihood of breast cancer patients to contract radiationinduced diseases which might involve heart, lung, liver and esophagus. Ultimately, the future clinical applications that emanate from this study may increase the life span of the breast cancer patients.

TABLE I

THE CALCUlATED SER FOR DIFFERENT CONCENTRATIONS OF 60 NM OF BIONPS IRRADIATED WITH PHOTON AND ELECTRON BEAMS.

\begin{tabular}{|c|c|c|c|}
\hline $\begin{array}{c}\text { Types of } \\
\text { Beam }\end{array}$ & Energy & $\begin{array}{c}\text { BiONPs } \\
\text { Concentration }(\mu \mathrm{M})\end{array}$ & SER \\
\hline \multirow{8}{*}{ Photon } & \multirow{4}{*}{$6 \mathrm{MV}$} & Control & 1.00 \\
\hline & & 0.05 & 1.07 \\
\hline & & 0.25 & 1.42 \\
\hline & & 0.50 & 1.88 \\
\hline & \multirow{4}{*}{$10 \mathrm{MV}$} & Control & 1.00 \\
\hline & & 0.05 & 1.45 \\
\hline & & 0.25 & 1.66 \\
\hline & & 0.50 & 2.29 \\
\hline \multirow{8}{*}{ Electron } & \multirow{4}{*}{$6 \mathrm{MeV}$} & Control & 1.00 \\
\hline & & 0.05 & 1.13 \\
\hline & & 0.25 & 1.33 \\
\hline & & 0.50 & 1.42 \\
\hline & \multirow{4}{*}{$12 \mathrm{MeV}$} & Control & 1.00 \\
\hline & & 0.05 & 1.05 \\
\hline & & 0.25 & 1.13 \\
\hline & & 0.50 & 1.22 \\
\hline
\end{tabular}

2) ROS Measurement induced by Radiation: Ionizing radiations are expected to disrupt the cancer cells progression either by direct radiation energy or by the mechanism of ROS indirectly [45]. Fig. 5 illustrates the intracellular ROS generation due to irradiation with photon and electron beams in the presence of different concentrations of BiONPs at 2,4 and 8 Gy of radiation dose.

The percentage of ROS generated by all doses of photon and electron beams together with all concentrations of BiONPs are near and above $100 \%$ in comparison to control without BiONPs. The highest concentration of BiONPs could commence the highest sensitization enhancement and elicited a higher percentage of ROS, as supported by a previous research work [18]. However, some irregularities are shown in Fig. 5 (A) and (D) in which the induction of ROS level after the irradiation were slightly lower than $100 \%$ when treated with a dose of 2 Gy and 4 Gy.

The typical ROS level in cancer cells is stated to be in a higher amount than ROS in normal cells [45]. The appearance of other elements such as NPs and radiation ions could have boosted or reduced the intracellular ROS level. It had been established that radiotherapy on cancer cells would yield more ROS and subsequently damage the DNA of the cancer cells.[45]. 

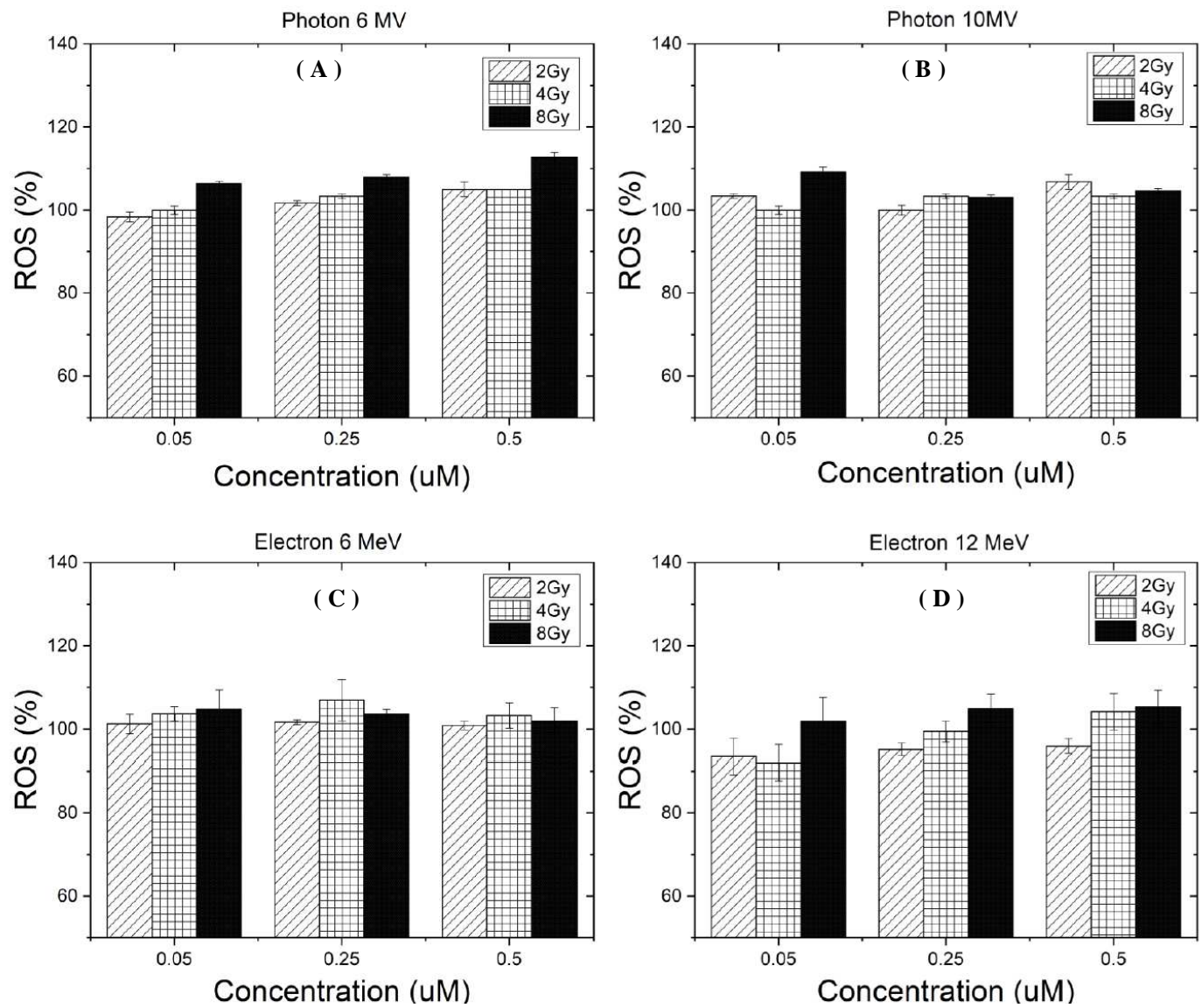

Fig. 5 Generation of intracellular ROS within the MCF-7 cells when treated with $60 \mathrm{~nm}$ of BiONPs irradiated with (A) 6 MV and (B) 10 MV photon beam, as well as (C) $6 \mathrm{MeV}$ and (D) $12 \mathrm{MeV}$ electron beam. Each error bar represents the standard deviation (SD).

Besides, in the presence of NPs, most of the radiation energy is hypothesized to be absorbed into the cells by the NPs [6], [18]. As one of the high atomic $Z$ elements, BiONPs could promote the photoelectric effects during the radiation, which in return produced a more elevated amount of ROS, leading towards increment of ROS and the cells impairments [18], [58]. Apart from that, there will be a minority of electrons that would evade the NPs absorption, further reacted with the surrounding and formed more ROS [18].

In this present study, it is assumed that the BiONPs that were internalized would also absorb the majority of the radiation dose. Then, the radiation interaction which was predominantly Compton effect was triggered. ROS will be generated and interact with the cancer cells' DNA and hence cause damage. Results of the ROS measurements were attuned with the SER value attained in Table 1.

\section{CONCLUSIONS}

This study highlights the importance of biological characteristics such as cytotoxicity, cellular uptake and ROS generation of rod-shaped BiONPs as a means to fully develop their properties as an alternative radiosensitizer. The current radiosensitizers used in radiotherapy are known to cause several side effects on healthy cells. Apart from being a high $\mathrm{Z}$ material, the BiONPs are found to be not toxic especially for $60 \mathrm{~nm}$ diameter size BiONPs. The BiONPs are also found to be suitable as a radiosensitizer for clinical radiotherapy on breast cancer cells as the results show the enhancement of radiosensitization and ROS generation induced by the BiONPs, which could increase the radiotherapy efficiency for the cancer treatment. Further investigation using various type of beam quality is required to fully understand the application of BiONPs in clinical settings.

\section{ACKNOWLEDGMENT}

This work was supported by Research University Grant (RUI: 1001/PPSK/8012212).

\section{REFERENCES}

[1] B. Bhushan, "Introduction to Nanotechnology," in Springer Handbook of Nanotechnology, B. Bhushan, Ed. Springer-Verlag Berlin Heidelberg, 2010, pp. 1-13.

[2] K. K. Jain, The Handbook of Nanomedicine, 3rd ed. Humana Press, 2008.

[3] L. Cui, "Optimization of Gold Nanoparticle Radiosensitizers for Cancer Therapy Optimization of Gold Nanoparticle Radiosensitizers," University of Toronto, 2016.

[4] S. Alarifi, D. Ali, S. Alkahtani, and M. S. Alhader, "Iron oxide nanoparticles induce oxidative stress, DNA damage, and caspase activation in the human breast cancer cell line," Biol. Trace Elem. Res., vol. 159, no. 1-3, pp. 416-424, 2014.

[5] B. Liu, L. Ezeogu, L. Zellmer, B. Yu, N. Xu, and D. Joshua Liao, "Protecting the Normal in Order to Better Kill the Cancer.," Cancer Med., vol. 4, no. 9, pp. 1394-403, 2015.

[6] W. N. Rahman, S. Corde, N. Yagi, S. A. Abdul Aziz, N. Annabell, 
and M. Geso, "Optimal Energy for Cell Radiosensitivity Enhancement by Gold Nanoparticles using Synchrotron-based Monoenergetic Photon Beams," Int. J. Nanomedicine, vol. 9, no. 1, pp. 2459-2467, 2014.

[7] L. Cui, S. Her, M. Dunne, G. R. Borst, R. D. Souza, R. G. Bristow, D. A. Jaffray, C. Allen, "Significant Radiation Enhancement Effects by Gold Nanoparticles in Combination with Cisplatin in Triple Negative Breast Cancer Cells and Tumor Xenografts," Radiat. Res., vol. 187, no. 2, pp. 147-160, 2017.

[8] M. J. Eblan and A. Z. Wang, "Improving chemoradiotherapy with nanoparticle therapeutics." Transl. Cancer Res., vol. 2, no. 4, pp. 320-329, 2013.

[9] J. J. Zhu, J. J. Shan, L. B. Sun, and W. S. Qiu, "Study of the radiotherapy sensitization effects and mechanism of capecitabine (Xeloda) against non-small-cell lung cancer cell line A549," Genet. Mol. Res., vol. 14, no. 4, pp. 16386-16391, 2015.

[10] W. D. Oliver, A. P. Duffy, and P. F. Hausner, "Case report of capecitabine toxicity and use of uridine triacetate," J. Oncol. Pharm. Pract., vol. 25, no. 2, pp. 470-473, 2019.

[11] L. Fernandez, A. Dominguez, W. Martinez, F. Sanabria, C. S. Leib, and B. R. G. in Thorax., "Pulmonary Toxicity Due to 5-Fluorouracil (5-FU) Manifested as Diffuse Alveolar Hemorrhage: Case Report," in D34. Lung Transplant and Drug Induced Lung Disease: Case Reports, 2018.

[12] H. Yang, C. Liu, D. Yang, H. Zhang, and Z. Xi, "Comparative study of cytotoxicity, oxidative stress and genotoxicity induced by four typical nanomaterials: The role of particle size, shape and composition," J. Appl. Toxicol., vol. 29, no. 1, pp. 69-78, 2009.

[13] A. L. Vega-Jiménez et al., "Bismuth subsalicylate nanoparticles with anaerobic antibacterial activity for dental applications," Nanotechnology, vol. 28, no. 43, 2017.

[14] S. Jain, B. Ch, J. A. Coulter, D. Ph, A. R. Hounsell, and T. Karl, "Cell-Specific Radiosensitization by Gold Nanoparticles at egavoltage Radiation Energies," Int J Radiat Oncol Biol Phys., vol. 79, no. 2, pp. 531-539, 2011.

[15] W. N. W. A. Rahman, R. A. Rashid, M. Muhammad, N. Dollah, K. A. Razak, and M. Geso, "Dose Enhancement by Different Size of Gold Nanoparticles Under Irradiation of Megavoltage Photon Beam," J. Sains Nukl. Malaysia, vol. 30, no. 2, pp. 23-29, 2018.

[16] C. Yang, K. Bromma, W. Sung, J. Schuemann, and D. Chithrani, "Determining the radiation enhancement effects of gold nanoparticles in cells in a combined treatment with cisplatin and radiation at therapeutic megavoltage energies," Cancers (Basel)., vol. 10, no. 150, pp. 1-16, 2018 .

[17] E. Shahhoseini, P. Ramachandran, W. R. Patterson, and M. Geso, "Determination of dose enhancement caused by AuNPs with Xoft ${ }^{\circledR}$ Axxent (B) Electronic ( $\mathrm{eBx}^{\mathrm{TM}}$ ) and conventional brachytherapy: in vitro study," Int. J. Nanomedicine, vol. 2018, no. 13, pp. 5733-5741, 2018

[18] S. J. Seo, S. M. Han, J. H. Cho, K. Hyodo, A. Zaboronok, H. You, K. Peach, M. A. Hill, J. K. Kim, "Enhanced production of reactive oxygen species by gadolinium oxide nanoparticles under core-innershell excitation by proton or monochromatic X-ray irradiation: implication of the contribution from the interatomic de-excitationmediated nanoradiator effec," Radiat. Environ. Biophys., vol. 54, no. 4, pp. 423-431, 2015.

[19] R. M. Lazim, R. A. Rashid, B. T. T. Pham, B. S. Hawkett, M. Geso, and W. N. Rahman, "Radiation Dose Enhancement Effects of Superparamagnetic Iron Oxide nanoparticles to the T24 Bladder Cancer Cell Lines Irradiated with Megavoltage Photon Beam Radiotheray," J. Sains Nukl. Malaysia, vol. 30, no. 2, pp. 30-38, 2018.

[20] M. Algethami, B. Feltis, and M. Geso, "Bismuth Sulfide Nanoparticles as a Complement to Traditional Iodinated Contrast Agents at Various X-Ray Computed Tomography Tube Potentials," $J$. Nanomater. Mol. Nanotechnol., vol. 06, no. 04, pp. 2-10, 2017.

[21] S. Tamilvanan, G. Gurumoorthy, S. Thirumaran, and S. Ciattini, "Synthesis, characterization, cytotoxicity and antimicrobial studies on $\mathrm{Bi}(\mathrm{III})$ dithiocarbamate complexes containing furfuryl group and their use for the preparation of Bi2O3nanoparticles," Polyhedron, vol. 121, pp. 70-79, 2017.

[22] V.A. Ovsyannikov, M.V. Zamoryanskaya, A.V. Semencha, K.A. Lycheva, T.S. Kol'tsova, O.V. Tolochko, and L.N. Blinov, "Development of bismuth oxide-based nanopreparation for the destruction of malignant neoplasms: Theoretical prerequisites, challenges, and practical approaches," Glas. Phys. Chem., vol. 41, no. 5, pp. 533-536, 2015.
[23] C. Stewart, K. Konstantinov, S. McKinnon, S. Guatelli, M. Lerch, A Rosenfeld, M. Tehei and S. Corde, "First proof of bismuth oxide nanoparticles as efficient radiosensitisers on highly radioresistant cancer cells," Phys. Medica, vol. 32, no. 11, 2016.

[24] M. Abudayyak, E. Öztaş, M. Arici, and G. Özhan, "Investigation of the toxicity of bismuth oxide nanoparticles in various cell lines," Chemosphere, vol. 169, pp. 117-123, 2017.

[25] N. A. M. Nor, Z. Mohd, H. R. A. Razak, Z. Eshak, and W. M. M Saad, "Synthetisation temperature-dependent cytotoxicity of bismuth oxide nanoparticles in vitro," Pertanika J. Sci. Technol., vol. 25, no. S, pp. 227-236, 2017.

[26] C. A. C. Stewart, "An investigation into the tailoring of bismuth oxide nanoceramic with a biomedical application as a high Z radiation enhancer for cancer therapy," University of Wollongong, 2014.

[27] M. Alqathami, A. Blencowe, M. Geso, and G. Ibbott, "Quantitative 3D determination of radiosensitization by bismuth-based nanoparticles," J. Biomed. Nanotechnol., vol. 12, no. 3, pp. 464-471, 2016.

[28] E. Taha, F. Djouider, and E. Banoqitah, "Monte Carlo simulations for dose enhancement in cancer treatment using bismuth oxide nanoparticles implanted in brain soft tissue," Australas. Phys. Eng. Sci. Med., vol. 41, pp. 363-370, 2018.

[29] S. Bancos, D. L. Stevens, and K. M. Tyner, "Effect of silica and gold nanoparticles on macrophage proliferation, activation markers, cytokine production, and phagocytosis in vitro," Int. J. Nanomedicine, vol. 10, pp. 183-206, 2015.

[30] C. Boyoglu, Q. He, G. Willing, S. Boyoglu-Barnum, V. A. Dennis, S. Pillai, and S. R. Singh, "Microscopic Studies of Various Sizes of Gold Nanoparticles and Their Cellular Localizations," ISRN Nanotechnol., vol. 2013, pp. 1-13, 2013.

[31] T. H. Kim, M. Kim, H. S. Park, U. S. Shin, M. S. Gong, and H. W. Kim, "Size-dependent cellular toxicity of silver nanoparticles," $J$. Biomed. Mater. Res. - Part A, vol. 100A, pp. 1033-1043, 2012.

[32] Z. A. Zulkifli, K. A. Razak, and W. N. W. A. Rahman, "The effect of reaction temperature on the particle size of bismuth oxide nanoparticles synthesized via hydrothermal method," AIP Conf. Proc. 1901, vol. 020007, pp. 1-5, 2018.

[33] Z. A. Zulkifli, K. A. Razak, W. N. W. A. Rahman, and S. Z. Abidin, "Synthesis and Characterisation of Bismuth Oxide Nanoparticles using Hydrothermal Method: The Effect of Reactant Concentrations and application in radiotherapy," J. Phys. Conf. Ser., vol. 1082, no. 1, 2018.

[34] Y. Li, X. Tian, Z. Lu, C. Yang, G. Yang, X. Zhou, H. Yao, Z. Zhu, Z. $\mathrm{Xi}$ and $\mathrm{X}$. Yang, "Mechanism for $\alpha-\mathrm{MnO} 2$ Nanowire-Induced Cytotoxicity in Hela Cells," J. Nanosci. Nanotechnol., vol. 10, no. 1, pp. 397-404, 2010.

[35] Y. Ibuki and T. Toyooka, "Nanoparticle Uptake Measured by Flow Cytometry," in Nanotoxicity Methods and Protocols, J. Reineke, Ed. Humana Press, 2012.

[36] C. Hanley, A. Thurber, C. Hanna, A. Punnoose, J. Zhang, and D. G Wingett, "The influences of cell Type and $\mathrm{ZnO}$ nanoparticle size on immune cell cytotoxicity and cytokine induction," Nanoscale Res. Lett., vol. 4, no. 12, pp. 1409-1420, 2009.

[37] D. Hernandez-Patlan, B. Solis-Cruz, A. Mendez-Albores, J. D. Latorre, X. Hernandez-Velasco, G. Tellez, and R. Lopez-Arellano, "Comparison of PrestoBlue (®) and plating method to evaluate antimicrobial activity of ascorbic acid, boric acid and curcumin in an in vitro gastrointestinal model," J. Appl. Microbiol., vol. 124, no. 2 , pp. 423-430, 2018.

[38] M. Xu, D. J. McCanna, and J. G. Sivak, "Use of the viability reagent PrestoBlue in comparison with alamarBlue and MTT to assess the viability of human corneal epithelial cells," J. Pharmacol. Toxicol. Methods, vol. 71, pp. 1-7, 2015.

[39] S. Gaucher and M. Jarraya, "Technical note: comparison of the PrestoBlue and LDH release assays with the MTT assay for skin viability assessment," Cell Tissue Bank., vol. 16, no. 3, pp. 325-329, 2015.

[40] M. Boncler, M. Rózalski, U. Krajewska, A. Podswdek, and C. Watala, "Comparison of PrestoBlue and MTT assays of cellular viability in the assessment of anti-proliferative effects of plant extracts on human endothelial cells," J. Pharmacol. Toxicol. Methods, vol. 69, no. 1, pp. 9-16, 2014.

[41] M. Sonnaert, I. Papantoniou, F. P. Luyten, and J. Schrooten, "Quantitative Validation of the Presto Blue ${ }^{\mathrm{TM}}$ Metabolic Assay for Online Monitoring of Cell Proliferation in a 3D Perfusion Bioreactor System," Tissue Eng. Part C Methods, vol. 21, no. 6, pp. 519-529, 
2014

[42] C. Zhu, W. Hu, H. Wu, and X. Hu, "No evident dose-response relationship between cellular ROS level and its cytotoxicity - A paradoxical issue in ROS-based cancer therapy," Sci. Rep., vol. 4, no. 5029, pp. 1-10, 2014.

[43] V. Jamier, L. A. Ba, and C. Jacob, "Selenium- and telluriumcontaining multifunctional redox agents as biochemical redox modulators with selective cytotoxicity," Chem. - A Eur. J., vol. 16, no. 36, pp. 10920-10928, 2010.

[44] E. L. H. Tang, J. Rajarajeswaran, S. Y. Fung, and M. S. Kanthimathi, "Antioxidant Activity of Coriandrum sativum and Protection Against DNA Damage and Cancer Cell Migration," BMC Complement. Altern. Med., vol. 13, no. 347, pp. 1-13, 2013.

[45] L. Tong, C. Chuang, S. Wu, and L. Zuo, "Reactive oxygen species in redox cancer therapy," Cancer Lett., vol. 07, no. 008, 2015.

[46] M. V. D. Z. Park, A. M. Neigh, J. P. Vermeulen, L. J. J. de la Fonteyne, H. W. Verharen, J. J. Briede, H. van Loveren, and W. H. de Jong, "The effect of particle size on the cytotoxicity, inflammation, developmental toxicity and genotoxicity of silver nanoparticles," Biomaterials, vol. 32, no. 36, pp. 9810-9817, 2011.

[47] H. Turkez and F. Geyikoglu, "The efficiacy of bismuth subnitrate against genotoxicity and oxidative stress induced by aluminum sulphate," Toxicol. Ind. Health, vol. 27, no. 2, pp. 133-142, 2011.

[48] M. Ahamed, M. J. Akhtar, M. A. M. Khan, S. A. Alrokayan, and H. A. Alhadlaq, "Oxidative stress mediated cytotoxicity and apoptosis response of bismuth oxide (Bi2O3) nanoparticles in human breast cancer (MCF-7) cells," Chemosphere, vol. 216, pp. 823-831, 2019.

[49] A. Salvati, I. Nellissen, A. Hasae, C. Aberg, S. Moya, A. Jacobs, F. Alnasser, T. Bewersdorff, S. Deville, A. Luch, and K. A. Dawson, "Quantitative measurement of nanoparticle uptake by flow cytometry illustrated by an interlaboratory comparison of the uptake of labelled polystyrene nanoparticles," NanoImpact, vol. 9, no. September 2017, pp. 42-50, 2018.

[50] A. Jochums, E. Friehs, F. Sambale, A. Lavrentieva, D. Bahnemann, and T. Scheper, "Revelation of Different Nanoparticle-Uptake
Behavior in Two Standard Cell Lines NIH/3T3 and A549 by Flow Cytometry and Time-Lapse Imaging," Toxics, vol. 5, no. 3, p. 15, 2017.

[51] H. Yang, Q. Y. Wu, C.S. Lao, M. Y. Li, Y. Gao, Y. Zheng, and B. Shi, "Cytotoxicity and DNA damage in mouse macrophages exposed to silica nanoparticles," Genet. Mol. Res., vol. 15, no. 3, pp. 1-14, 2016.

[52] I. L. Hsiao, F. S. Bierkandt, P. Reichardt, A. Luch, Y-J. Huang, N. Jakubowski, J. Tentschert, and A. Haase, "Quantification and visualization of cellular uptake of $\mathrm{TiO} 2$ and $\mathrm{Ag}$ nanoparticles: Comparison of different ICP-MS techniques," J. Nanobiotechnology, vol. 14, no. 1, pp. 1-13, 2016.

[53] B. D. Chithrani, A. A. Ghazani, and W. C. W. Chan, "Determining the Size and Shape Dependence of Gold Nanoparticles Uptake Into Mammalian Cells," Nano Lett., vol. 6, no. 4, pp. 662-668, 2006.

[54] P. V Asharani, N. Xinyi, M. P. Hande, and S. Valiyaveettil, "DNA damage and p53-mediated growth arrest in human cells treated with platinum nanoparticles." Nanomedicine, vol. 5, no. 1, pp. 51-64, 2010.

[55] R. A. Rashid, N. Dollah, R. Abdullah, and W. N. W. A. Rahman, "The effects of wound dressings on the dose at surface and depth of maximum dose (dmax) for photon and electron beam radiotherapy," J. Med. Phys. Biophys., vol. 4, no. 1, pp. 103-107, 2017.

[56] W. N. W. A. Rahman, "Gold nanoparticles: novel radiobiological dose enhancement studies for radiation therapy, synchrotron based microbeam and stereotactic radiotherapy," RMIT University, 2010.

[57] J. C. L. Chow, "Photon and electron interactions with gold nanoparticles: A Monte Carlo study on gold nanoparticle-enhanced radiotherapy," in Nanobiomaterials in Medical Imaging: Applications of Nanobiomaterials, Elsevier Inc., 2016, pp. 45-70.

[58] A. Detappe, S. Kunjachan, P. Drane, S. Kotb, M. Myronakis, D. E. Biancur, T. Ireland, M. Wagar, F. Lux, O. Tillement, and R. Berbeco, "Key clinical beam parameters for nanoparticle-mediated radiation dose amplification," Nat. Sci. Reports, vol. 6, no. 34040, pp. 1-8, 2016. 